INPLASY

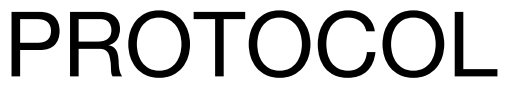

To cite: Chen et al. Efficacy and Safety of Acupuncture at Renying Point(ST9) in Treating for Essential Hypertension: a protocol for systematic review and meta-analyses. Inplasy protocol 202150040. doi: 10.37766/inplasy2021.5.0040

Received: 11 May 2021

Published: 11 May 2021

Corresponding author: Dandan Chen

819328570@qq.com

Author Affiliation:

Fenghua District Hospital of Traditional Chinese Medicine, Ningbo City, Zhejiang

Province.

Support: None.

Review Stage at time of this submission: Preliminary searches.

Conflicts of interest: None declared.

\section{Efficacy and Safety of Acupuncture at Renying Point(ST9) in Treating for Essential Hypertension: a protocol for systematic review and meta-analyses}

Chen, DD1; Hou, XB2; Cheng, TD3; Wang, D4; Dai, XJ5; Wang, Y6; Cui, BX7; Wang YY8; Xu, H9; Chen, HZ10; Shen, PF11.

Review question / Objective: To evaluate the safety and effectiveness of acupuncture at Renying acupoint in the treatment of essential hypertension.

Condition being studied: Hypertension is a vascular synthesis characterized by continuous increase in arterial blood pressure. Symptoms are independent risk factors that cause damage to important target organs such as heart, brain, and kidneys. Hypertension has become a major public health problem worldwide. Therefore, safe, effective and stable blood pressure reduction is the key to the prevention and control of major cardiovascular and cerebrovascular events. Due to the interaction of clinical and western medicine, its contraindications, and the limitation of adverse reactions It is necessary to find a method that can be used for a long time and is safe and effective to reduce blood pressure and significantly improve A new approach to prognosis. Acupuncture is widely used in the treatment of hypertension in China, and a large number of related studies have been published, and the results show that the effect is definite.

INPLASY registration number: This protocol was registered with the International Platform of Registered Systematic Review and Meta-Analysis Protocols (INPLASY) on 11 May 2021 and was last updated on 11 May 2021 (registration number INPLASY202150040).

\section{INTRODUCTION}

Review question / Objective: To evaluate the safety and effectiveness of acupuncture at Renying acupoint in the treatment of essential hypertension.
Condition being studied: Hypertension is a vascular synthesis characterized by continuous increase in arterial blood pressure. Symptoms are independent risk factors that cause damage to important target organs such as heart, brain, and kidneys. Hypertension has become a major public health problem worldwide. 
Therefore, safe, effective and stable blood pressure reduction is the key to the prevention and control of major cardiovascular and cerebrovascular events. Due to the interaction of clinical and western medicine, its contraindications, and the limitation of adverse reactions It is necessary to find a method that can be used for a long time and is safe and effective to reduce blood pressure and significantly improve A new approach to prognosis. Acupuncture is widely used in the treatment of hypertension in China, and a large number of related studies have been published, and the results show that the effect is definite.

\section{METHODS}

Participant or population: Patients with essential hypertension.

Intervention: Acupuncture at Renying Point.

Comparator: Other treatment options that exclude acupuncture.

Study designs to be included: RCTs.

Eligibility criteria: There are clear and recognized diagnostic criteria and efficacy criteria, regardless of gender, age, and origin of the case.

Information sources: The two reviewers independently collected data on acupuncture at renying point(ST9) in treating for essential hypertension from CNKI, Wanfang, VIP, Chinese Biomedical Literature Database, Cochrane Library, Pubmed, Embase, CINAHL, Ovidweb, Web of science, from their inception to May 2021. All randomized controlled trials (RCTs) without language restriction were considered for inclusion.

Main outcome(s): Treatment efficacy.

Additional outcome(s): Adverse reactions, Systolic blood pressure, Diastolic blood pressure, Pulse pressure difference.
Data management: Data extraction and analysis will be performed independently by two researchers. When differences and opinions are inconsistent, they should be resolved through discussion. If the differences encountered cannot be resolved through discussion, a third author will be invited to resolve them.

Quality assessment / Risk of bias analysis: We will use the "deviation risk" tool in Cochrane Manual v.5.1.0 to assess the deviation risk of each article in the literature. Assessments include sequence generation, allocation sequence hiding, blindness of participants and people, and outcome evaluators, incomplete outcome data, selective outcome reporting and other sources of bias. If the risk of bias is high in the literature, we will try to explain and discuss the causes of bias.

Strategy of data synthesis: For dichotomous data, we will denote the outcomes as relative risks (RRs) with $95 \%$ Cls. If the $I^{2}$ test is less than $50 \%$, the fixed effects model will be used for data synthesis. If the $\mathrm{I}^{2}$ test is between $\mathbf{5 0} \%$ and $75 \%$, the random-effects model will be conducted for data synthesis. If the $I^{2}$ test is higher than $75 \%$, we will investigate possible reasons from both clinical and methodological perspectives, and provide a descriptive analysis or conduct subgroup analysis. For continuous data, if no heterogeneity is detected, we will use mean difference (MD) or standard MD (SMD) to measure the therapeutic effect of $95 \%$ Cls. If significant heterogeneity is found, we will use the random-effects model instead.

Subgroup analysis: If there are obvious heterogeneity, we will conduct subgroup analysis to identify the sources of heterogeneity. We will conduct subgroup analysis according to different course time or different outcome indicators.

Sensitivity analysis: When sufficient trials are available, sensitivity analysis will be performed by sequentially eliding each trial to check the robustness of the final results. 
Country(ies) involved: China.

Keywords: Acupuncture; Renying Point;

Essential Hypertension; Protocol.

Contributions of each author:

Author 1 - Dandan Chen.

Email: 819328570@qq.com

Author 2 - Xianbing Hou.

Email: houxianbing007@126.com

Author 3 - Tongfei Cheng.

Email: 20964891@qq.com

Author 4 - Dan Wang.

Email: 82537493@qq.com

Author 5 - Xiaojun Dai.

Email: 2398431959@qq.com

Author 6 - Yao Wang.

Email: wangy198302@sohu.com

Author 7 - Bixian Cui.

Email: 459397003@qq.com

Author 8 - Yuanyuan Wang.

Email: wagnyy9208@163.com

Author 9 - Hui Xu.

Email: 598702325@qq.com

Author 10 - Hongzhou Chen.

Email: 2352326376@qq.com

Author 11 - Pengfei Shen.

Email: shenpengfei001@126.com 